\title{
ON THE HAHN-BANACH EXTENSION PROPERTY
}

\author{
JONATHAN M. BORWEIN ${ }^{1}$
}

\begin{abstract}
A self-contained and brief proof is given of the equivalence of the Hahn-Banach extension property (HB) and the conditional order completeness of the range space (LUB). Various other equivalences are discussed.
\end{abstract}

1. Introduetion. Let $Y$ be an ordered vector space. This is to say that $Y$ is a real vector space endowed with an associated partial order induced by a convex cone $S$ (containing zero) by $x_{1} \geq x_{2}$ if $x_{1}-x_{2}$ lies in $S$. Recall that $Y$ is said to be conditionally order complete or to have the least upper bound property (LUB) if every nonempty subset $A$ of $Y$ with an upper bound has a least upper bound, $\sup A$ [4]. Since $\geq$ is antisymmetric only when $S$ is pointed, these suprema are generally not unique.

Now $Y$ is said to have the Hahn-Banach extension property (HB) if the following holds. Suppose that $X$ is a real vector space and that $p: X \rightarrow Y$ is a sublinear operator (with respect to $S$ ). Let $M$ be a vector subspace of $X$ and let $T: M \rightarrow Y$ be linear with $T m \leq p(m)$ for all $m$ in $M$. Then there exists a linear operator $T_{0}: X \rightarrow Y$ extending $T$ and dominated by $p$ on $X$ [4]. If the extension is only asserted to exist when $M$ is a hyperplane we say $Y$ has the Hahn-Banach extension property for hyperplanes. Suppose that $Y$ is a topological vector space while $X$ is constrained to lie in some subclass of topological vector spaces $C$ (for example, Banach spaces or $l_{1}$ spaces). We say that $Y$ has the continuous Hahn-Banach property for $C$ if extensions exist for continuous linear operators dominated on closed subspaces by continuous sublinear operators between $X$ and $Y$.

That (HB) and (LUB) are equivalent was originally shown by Silverman and Yen $[12,4]$, assuming that $S$ is linearly closed. That linear closure follows from (LUB) is straightforward. Much less immediate was the proof that (HB) implies linear closure, due to Bonnice and Silverman $[1,2]$.

Their arguments were incomplete and were finally made adequate by To [13]. These arguments, while geometric, are lengthy ad involve considrable case analysis. Elster and Nehse $[5,6]$, using the Bonnice-Silverman-To-Yen proof as a basis, have shown the equivalence of a variety of other convex optimization and positive operator theoretic results.

Recently, Ioffe [9] has established the equivalence of (HB) and (LUB) as a consequence of a more general result on linear selections for fans $[8,9]$. In this note we wish to show that, using the same vector space, same hyperplane and essentially the

Received by the editors August 24, 1981 and, in revised form, November 30, 1981.

1980 Mathematics Subject Classification. Primary 46A40, 46A55, 06F20.

Key words and phrases. Ordered vector space, Hahn-Banach extension property, least upper bound, Lagrange multiplier theorem.

${ }^{1}$ Research partially supported by NSERC Grant A5116. 
same sublinear functional as were used in [9], it is possible to give a much simpler proof.

\section{The central result.}

THEOREM 1. An ordered vector space with the Hahn-Banach extension property for hyperplanes has the least upper bound property.

Proof. Let $A$ be a nonempty subset of $Y$ with an upper bound. Let $B$ denote the nonempty set of upper bounds in $Y$ for $A$. Let us list $A$ and $B$ as

$$
A:=\{a(i) \in Y \mid i \in I\}, \quad B:=\{b(i) \in Y \mid i \in I\}
$$

for some index set $I$, possibly with repetitions. Let $X$ be the direct sum of $I$ copies of R. Equivalently, $X$ is the vector space of all real valued functions on $I$ which have finite support. Now define $p: X \rightarrow Y$ by

$$
\begin{aligned}
p(x) & :=\sum_{I}\left[x^{+}(i) b(i)-x^{-}(i) a(i)\right] \\
& =\sum_{I} x^{+}(i)[b(i)-a(i)]+\sum_{I} x(i) a(i) .
\end{aligned}
$$

Here $x^{+}$is the positive part of $x$ and $x^{-}$is the negative part [11]. Since $x$ has finite support, $p$ is well defined. Now (2.2) shows that $p$ is sublinear. Indeed, since $x^{+}(i)=\max \{x(i), 0\}$ and $b(i)-a(i) \geq 0, x^{i}(i)[b(i)-a(i)]$ is sublinear and it follows that $p$ is. Let $\Phi$ be the linear functional on $X$ given by

$$
\Phi(x):=\sum_{I} x(i)
$$

Suppose that $\Phi(x)=0$. Then one has $\sum_{I} x^{+}(i)=\sum_{I} x^{-}(i)$. It follows that $\sum x^{+}(i) b(i) \geq \sum x^{-}(i) a(i)$ since every member of the convex hull of $A$ is majorized by every member of the convex hull of $B$ because $B$ majorizes $A$. Thus we derive from (2.1) that

$$
\Phi(x)=0 \text { implies } p(x) \geq 0 .
$$

The Hahn-Banach extension property for hyperplanes may be applied to $p$, to $M:=$ $\varphi^{-1}\{0\}$ and $T:=0$. We are guaranteed a linear extension $T_{0}$ of $T$ with $T x \leq p(x)$. Let $e(i)$ denote the function in $X$ which is zero except at $i$ where it has value one. Then for $i, j$ in $I, e(i)-e(j)$ lies in $M$ and thus $c:=T e(i)=T e(j)$ is independent of $i$ in $I$. In addition

$$
a(j)=-p(-e(j)) \leq T e(j)=c=T e(i) \leq p(e(i))=b(i) .
$$

Since $i$ and $j$ are arbitrary this shows that $c$ majorizes $A$ and minorizes $B$. Thus $c$ is a least upper bound for $A$.

Theorem 1 is phrased as it is to make it apparent that the implication (HB) implies (LUB) uses no hidden transfinite arguments. Since the axiom of choice in conjunction with (LUB) yields (HB), exactly as in the scalar case $[3,4,11]$, we derive

COROLLARY 2. In the presence of the axiom of choice or appropriate maximality principle, $Y$ has the Hahn-Banach extension property (for hyperplanes) if and only if $Y$ has the least upper bound property. 
The other equivalences given in Day [4] and in Elster and Nehse [5, 6] now follow. In the next section we extend certain of these formulations.

3. Alternate formulations. We may suppose, on translation, that zero belongs to $A$. A moment's reflection on the proof of Theorem 1 shows that this allows us to replace $(2.4)$ by

$$
\Phi(x) \geq 0 \text { implies } p(x) \geq 0 .
$$

Trivially, Slater's condition [10] holds:

$$
\Phi(\hat{x})>0 \text { for some } \hat{x} \text { in } X .
$$

The Lagrange multiplier theorem (LM) in [3], or the Kuhn-Tucker theorem (KT) in [6], or the extended Farkas-Minkowski theorem (FM) in [6] insures the existence of a linear multiplier $\lambda: \mathbf{R} \rightarrow Y$ such that $\lambda\left(\mathbf{R}_{+}\right) \subset S$ and

$$
\lambda \Phi(x) \leq p(x),
$$

for all $x$ in $X$. As with (2.5) we derive

$$
a(j)=-p(-e(j)) \leq \lambda(1) \leq p(e(i)) \leq b(i)
$$

so that $\lambda(1) \in \sup A$.

Thus with the best possible regularity condition for the simplest possible constraint structure we obtain (LUB). Indeed, one has a single nontrivial linear inequality and a convex homogeneous objective function. As (KT), (LM) and (FM), for convex operators satisfying a very general regularity condition, all follow from (LUB), $[3,6]$ we have proved

THEOREM 3. Suppose (KT), (LM) or. (FM) holds for homogeneous convex optimization problems with one linear inequality constraint. Then $Y$ is conditionally order complete and $(K T),(L M)$ and $(F M)$ hold for all convex programming problems with the appropriate regularity condition.

REMARK. Suppose that one is given a sublinear operator $p: X \rightarrow Y$ and a point in $x_{0}$ in $X$, and one wishes to find a linear operator $T: X \rightarrow Y$ majorized by $p$ with $T x_{0}=p\left(x_{0}\right)$. Fel'dman [7] has shown that this can be achieved whenever $Y$ has the monotone chain property: every increasing chain in $Y$ with an upper bound has a supremum. Every closed finite dimensional cone has this property while only simplicial cones are order complete [7]. Thus this restricted extension property does not imply the full Hahn-Banach extension property. It follows that the existence of linear minorants (subgradients) does not imply that $Y$ is order complete.

4. A continuous result. In application one is often content to know that the continuous Hahn-Banach extension property holds for Banach spaces. We finish by showing that in many circumstances much less suffices to force $Y$ to be order complete.

THEOREM 4. Suppose $Y$ is a topological linear space and that the order in $Y$ is a normal lattice order with topologically complete order intervals. Suppose that $Y$ has the continuous Hahn-Banach extension property for $l_{1}$ spaces. Then $Y$ has the least upper bound property. 
Proof. Let $A$ and $B$ be as in Theorem 1. Let $X:=l_{1}(I)$, the space of all absolutely summable functions on $I$. Let $a$ be in $A$ and $b$ in $B$. Then $a(i) \vee a \leq$ $b(i) \wedge b$ for any $i$ in $I$. We first observe that for any $(c(i))$ with $a \leq c(i) \leq b$ and any $y$ in $l_{1}(I), \sum_{I} y(i) c(i)$ exists as a rearrangement invariant limit in $Y$. Indeed let $d:=|a| \vee|b|$ and $D:=[-d, d]$. Then, for any finite set $F$ in $I$,

$$
c_{F}:=\sum_{F} y(i) c(i) \in\left(\sum_{F}|y(i)|\right) D \text {. }
$$

Let $\epsilon>0$ be given and suppose $\sum_{i \in F}|y(i)|<\epsilon$. Then for $F_{1}, F_{2} \supset F$ one has

$$
c_{F_{1}}-c_{F_{2}} \in\left(\sum_{F_{1} \Delta F_{2}}|y(i)|\right) D \subset \epsilon D .
$$

Since $D$ is an order interval and $S$ is normal, $D$ is bounded and (4.1) and (4.2) shows that $\left(c_{F}\right)$ is a Cauchy net which lies in some multiple of $D$. As $D$ is complete $c_{F}$ converges to some point in $Y$ as required.

Now define $p: X \rightarrow Y$ by

$$
p(x):=\sum_{T}\left[x^{+}(i)(b(i) \wedge b)-x^{-}(i)(a(i) \vee a)\right]
$$

Then the previous observations show that $p$ is well defined. Moreover, $p$ is sublinear as the limit of sublinear operators of the general form in (2.1) and (2.2). Next, observe that for $x$ in the unit ball of $l_{1}(I),(4.1)$ implies that $p(x) \leq d$. Thus $p$ is bounded above on a neighbourhood of zero. Since the order is normal it follows that $p$ is continuous [3]. Now define $\Phi$ on $l_{1}(I)$ as in (2.3) and observe that $\Phi$ is continuous since $\Phi$ lies in $l_{\infty}(I)$. Since $\left\{x \in l_{1}(I) \mid \Phi(x)=0\right.$, supp $x$ is finite $\}$ is dense in $\Phi^{-1}\{0\}$ and since $p$ is continuous and $S$ is closed, (2.4) holds with $X=$ $l_{1}(I)$. The continuous extension property allows us to derive, as in Theorem 1 , the existence of $c$ in $Y$ with

$$
a(j) \leq a(j) \vee a \leq c \leq b(i) \wedge b \leq b(i)
$$

for $i, j$ in $I$. Thus $c \in \sup A$ and $Y$ is order complete.

Since any Fréchet lattice [11] has the properties required of $Y$ we have the following corollary.

COROLlaRY 5. A Fréchet lattice is order complete if and only if it has the continuous Hahn-Banach extension property for Banach spaces ( $l_{1}$ spaces).

It is interesting to extend this result to nonlattices. The lattice property is only used to allow us to replace $A$ and $B$ by subsets of an order interval. Now one may combine the argument of Theorem 4 with Steps 2 and 3 on p. 136 of [4]. This establishes Theorem 4 with deletion of "lattice" in the statement of that result.

\section{REFERENCES}

1. W. Bonnice and R. Silverman, The Hahn-Banach theorem for finite dimensional spaces, Trans. Amer. Math. Soc. 121 (1966), 210-222.

2. , The Hahn-Banach extension and the least upper bound properties are equivalent, Proc. Amer. Math. Soc. 18 (1967), 843-850.

3. J. M. Borwein, A Lagrange multiplier theorem and a sandwich theorem for convex relations, Math. Scand. (1981). 
4. M. M. Day, Normed linear spaces, Springer-Verlag, Berlin and New York, 1973.

5. K.-H. Elster and R. Nehse, Konjugierte operatoren und subdifferentiale, Math. Operationsforsch. Statist. 6 (1975), 641-657.

6. _ , Necessary and sufficient conditions for the order-completeness of partially ordered vector spaces, Math. Nachr. 81 (1981), 301-311.

7. M. M. Fel'dman, Sufficient conditions for the existence of supporting operators for sublinear operators, Sibirsk. Mat. Ż. 16 (1975), 132-138. (Russian)

8. A. D. Iofie, Nonsmooth analysis: Differential calculus of nondifferentiable mappings, Trans. Amer. Math. Soc. 266 (1981), 1-55.

9. $\ldots, A$ new proof of the equivalence of the Hahn-Banach extension and the least upper bound properties, Proc. Amer. Math. Soc. 82 (1981), 385-389.

10. D. G. Luenberger, Optimization by vector space methods, Wiley, New York, 1969.

11. A. L. Peressini, Ordered topological vector spaces, Harper \& Row, New York, 1967.

12. R. Silverman and Ti Yen, The Hahn-Banach theorem and the least upper bound property, Trans. Amer. Math. Soc. 90 (1959), 523-526.

13. T.-O. To, The equivalence of the least upper bound property and the Hahn-Banach extension property in ordered vector spaces, Proc. Amer. Math. Soc. 30 (1971), 287-296.

Department of Mathematics, Carnegie-Mellon University, Pittsburgh, PENNSYLVANIA 15213

Department of Mathematics, Dalhousie University, Halifax, Nova Scotia, CANADA (Current address) 